\title{
Role of gamma-delta T cells in host response against Staphylococcus aureus-induced pneumonia
}

Ping Cheng ${ }^{1 \dagger}$, Tao Liu ${ }^{1 \dagger}$, Wei-Ying Zhou ${ }^{2}$, Yuan Zhuang ${ }^{1}$, Liu-sheng Peng ${ }^{1}$, Jin-yu Zhang ${ }^{1}$, Zhi-Nan Yin ${ }^{3}$, Xu-hu Mao', Gang Guo ${ }^{1}$, Yun Shi ${ }^{1 *}$ and Quan-ming Zou' ${ }^{1 *}$

\begin{abstract}
Background: Staphylococcus aureus is the major cause of hospital-acquired and community-acquired pneumonia. Host defense to S.aureus infection is largely mediated by the innate immune system. $\gamma \delta$ T cells play an important role in innate immunity to many infectious diseases. However, less is known about the role of these cells during S.aureus-induced pneumonia. In this study, we examined the response and the role of $\gamma \delta T$ cells to pulmonary S.aureus infection.

Results: Mice infected with $S$. aureus intranasally showed rapid $\gamma \delta T$ cells accumulation in the lung. Deficiency of $\gamma \delta T$ cells led to attenuated bacterial clearance and less tissue damage in lung compared with WT mice. Moreover, TCR- $\delta^{-/-}$mice exhibited impaired neutrophil recruitment and reduced cytokine production at the site of infection. The $\gamma \delta T$ cells in response to pulmonary S. aureus infection mainly secreted IL-17 and $\gamma \delta T$ cells deficiency reduced IL-17 production, which might regulate the production of neutrophil-inducing cytokine/chemokine in the S. aureus-infected lungs.

Conclusions: Accumulation of $\gamma \delta T$ cells in the lungs to $S$. aureus infection is beneficial for bacteria clearance and also contributes to the tissue damage. These cells were the primary source of IL-17, which might influence the recruitment of neutrophils at the early stage of infection.
\end{abstract}

\section{Background}

Staphylococcus aureus (S.aureus) is a Gram-positive, extracellular bacterium that cause a variety of infections, including pneumonia, septic arthritis, bacteremia, endocarditic and cellulitis [1]. S.aureus-induced pneumonia accounts for $20-50 \%$ nosocomial pneumonia and $25.5 \%$ of community-acquired pneumonia, which causes especially severe pulmonary infection and is associated with high morbidity and mortality $[2,3]$. In recent years, the rapid emergence of Methicillin-resistant S.aureus raises great concern about the cellular and molecular mechanisms of host defense against S.aureus infection.

\footnotetext{
* Correspondence: shiyuntmmu@gmail.com; qmzou@mail.tmmu.com.cn ${ }^{\dagger}$ Equal contributors

${ }^{1}$ Department of Clinical Microbiology and Immunology, Faculty of Medical Laboratory Science, Third Military Medical University and National Engineering Technological Research Center of Immunological Biologicals, Chongqing 400038, China

Full list of author information is available at the end of the article
}

Innate immune response plays a critical role in the host defense against S.aureus infection. Rapid and massive neutrophil recruitment to the site of infection is essential for host defense against invading pathogenic microbes [4]. In a mouse model of S. aureus pneumonia, depletion of neutrophils resulted in delayed bacteria clearance and decreased survival of infected mice [5]. Following S.aureus challenge, alveolar macrophages and dendritic cells (DC) are also recruited to the site of infection and contribute to bacterial clearance, but might also exacerbate lung inflammation and injury [6,7]. Thus, early interactions between these innate cells and pathogens are important in determining the outcome of S.aureus-induced pneumonia.

In addition to these conventional innate cells, $\mathrm{T}$ cells bearing the $\gamma \delta$ TCR $(\gamma \delta$ T cells), another subset of innate immune lymphocytes, play an essential role in the mucosal host defense bacteria infection. Previous studies suggest that $\gamma \delta$ T cells play an important role in regulating

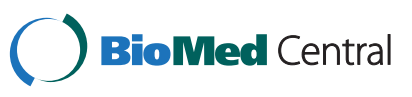


the initial immune response to lung pathogens via influencing the recruitment of neutrophils [8], DC or macrophages [9]. Although the lung $\gamma \delta$ T cells comprise a very small percentage of pulmonary immune cells [10], they seem to be the first line of immune response against invading microbial pathogens. Depletion of $\gamma \delta$ T cells led to impaired host defense to lung infections by Klebsiella pneumonia [11], Mycobacterium tuberculosis [12] or Streptococcus pneumoniae [13]. It has been reported that $\gamma \delta$ T cells also play an important role in cutaneous $S$. aureus infection [14]. However, the exact roles of these cells played in S.aureus-induced pneumonia have not been well defined.

In the present study, we first observed an accumulation of $\gamma \delta$ T cells in lungs infected with S. aureus. To investigate their role during an acute $S$. aureus pneumonia, we assessed the effects of the deficiency of $\gamma \delta \mathrm{T}$ cells on the host defense against S.aureus infection. Our results demonstrate that $\gamma \delta \mathrm{T}$ cell knock out attenuated bacterial clearance and resulted in less severe lung damage. Furthermore, a defect of IL-17 induction and impaired neutrophil recruitment following $S$. aureus challenge was observed in the absence of $\gamma \delta$ T cells. We presumed that the production of IL-17 by $\gamma \delta$ T cells had effects on early neutrophilmediated lung inflammation and acute tissue injury.

\section{Results}

\section{$\gamma \delta \mathrm{T}$ cells were accumulated in the lungs infected} with S. aureus

To investigate whether $\gamma \delta$ T cells are involved in host response to $S$. aureus infection, the kinetics of the $\gamma \delta$ T cell response in the lungs of mice infected with S.aureus was examined by flow cytometry. In mock-infected mice, $\gamma \delta \mathrm{T}$ cells comprised only about $0.5 \%$ of the lung $\mathrm{CD}^{+} \mathrm{T}$ lymphocytes, whereas the percentage was significantly increased from $6 \mathrm{~h}$ post-infection (Figure 1A). While similar kinetics was observed in the absolute numbers of $\gamma \delta \mathrm{T}$ cells in the total lung following $S$. aureus challenge

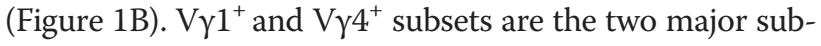
sets of lung $\gamma \delta$ T cells. To determine which subsets of pulmonary $\gamma \delta \mathrm{T}$ cells accumulated at the infected sites, we examined the proportion of ${\mathrm{V} \gamma 1^{+}}^{+}$and $\mathrm{V}_{\gamma} 4^{+} \gamma \delta$ T cells in the lungs at $6 \mathrm{~h}$ post-infection. As shown in Figure $1 \mathrm{C}$, $\mathrm{V} \gamma 1^{+}$cells (identified as $\mathrm{CD}^{+} \mathrm{TCR} \alpha \beta^{-} \mathrm{TCR} \gamma \delta^{+} \mathrm{V} 1^{+}$cells) were about $13.1 \pm 3.1 \%(n=6)$ of total $\gamma \delta^{+} T$ cells in mockinfected mice, and the proportion of ${\mathrm{V} \gamma 1^{+}}^{+}$cells was maintained following bacteria challenge. Nevertheless, considering the dramatically increased number of lung cells in response to acute bacterial infection, absolute numbers of $\mathrm{V} \gamma^{+}{ }^{+}$cell was significant increased at $6 \mathrm{~h}$ post-infection with S.aureus $\left(p<0.01\right.$ Figure 1D). $V \gamma 4^{+} \gamma \delta$ T cells (identified as $\mathrm{CD}^{+}{ }^{+} \mathrm{TCR} \alpha \beta^{-} \mathrm{TCR} \gamma \delta^{+} \mathrm{V}_{\gamma} 4^{+}$cells) comprised approximately $21.7 \pm 2.2 \%(\mathrm{n}=6)$ of $\gamma \delta^{+} \mathrm{T}$ cells in the mock-infected lung and were significantly increased in response to $S$. aureus challenge (mean $37.9 \pm 5.2 \%$ ). Consistently, their absolute number almost increased up to 6 -fold at $6 \mathrm{~h}$ post infection (Figure 1D). Thus, these results demonstrated that pulmonary $S$. aureus infection results in an accumulation of $\gamma \delta$ T-cell in the lung.

\section{Deficiency of $\gamma \delta$ T cells led to attenuated bacterial clearance but less acute severe lung damage}

In order to investigate the role of $\gamma \delta$ T cells in S. aureusinduced pneumonia, we next examined the effect of deficiency of $\gamma \delta$ T cells on the host response to S. aureus infection. Initially, we compared the bacterial clearance and morbidity of TCR- $\delta^{-/-}$mice and wild-type (WT) following an intra-nasal instillation of sub-lethal S.aureus infection $\left(5 \times 10^{8} \mathrm{CFU}\right)$. The bacterial burden in lungs and spleens at both 24 and 48 hours post-infection was significantly higher in TCR- $\delta^{-/-}$mice than that in WT mice (Figure 2A). Next, the survival rate of TCR- $\delta^{-/-}$ mice and WT mice after challenge with a lethal dose of S.aureus $\left(5 \times 10^{9} \mathrm{CFU}\right)$ was analyzed (Figure $\left.2 \mathrm{~B}\right)$. Both WT and TCR- $\delta^{-1-}$ mice started to die at 12 hours and no significantly different mortality rate was observed. However, there were significant differences in pulmonary histology between TCR- $\delta^{-1-}$ mice and WT mice. As a control, uninfected WT mice showed normal lung parenchyma. At 12 hours post-infection, the prominent destruction of alveolae and the infiltration of large amounts of inflammatory cells were observed in WT mice, whereas less immune cell infiltration was seen in TCR- $\delta^{-1-}$ mice (Figure 3A). Overall the severity of pneumonia in $\mathrm{TCR}-\delta^{-/-}$mice was significantly lower than that in WT mice (Figure 3B). These results demonstrate that the lack of $\gamma \delta \mathrm{T}$ cells led to attenuated bacterial clearance but less severe acute lung damage.

\section{Lung neutrophils infiltration in response to $S$. aureus} pulmonary infection was impaired in TCR- $\delta^{-/-}$mice

Neutrophils provide an essential defense against invading $S$. aureus [4]. To test whether neutrophils are involved in the attenuated bacterial clearance in TCR- $\delta^{-/-}$mice during S.aureus pneumonia, the neutrophils in the lungs at $6 \mathrm{~h}$ post-challenge were examined by flow cytometry. Neutrophils were identified by co-expression of CD11b and Gr-1. Compared with uninfected mice, infected WT mice exhibited a significant increase of $\mathrm{CD}_{11} \mathrm{~b}^{+} \mathrm{Gr}-1^{\text {high }}$ cells, whereas, neutrophils in infected TCR- $\delta^{-1-}$ mice were significantly lower than that in infected WT mice (Figure $4 \mathrm{~A} \& \mathrm{~B}$ ). Furthermore, the number of neutrophils $\left(\mathrm{CD} 11 \mathrm{~b}^{+} \mathrm{Gr}-\mathrm{1}^{\text {high }}\right)$ in BAL fluid of infected mice was examined. Fewer neutrophils were detected in the BAL fluid of TCR- $\delta^{-1-}$ mice as compared to wild-type mice (Figure 4C). These results suggest that the deletion of $\gamma \delta \mathrm{T}$ cell impair neutrophil recruitment to the lungs after infection with S.aureus. 

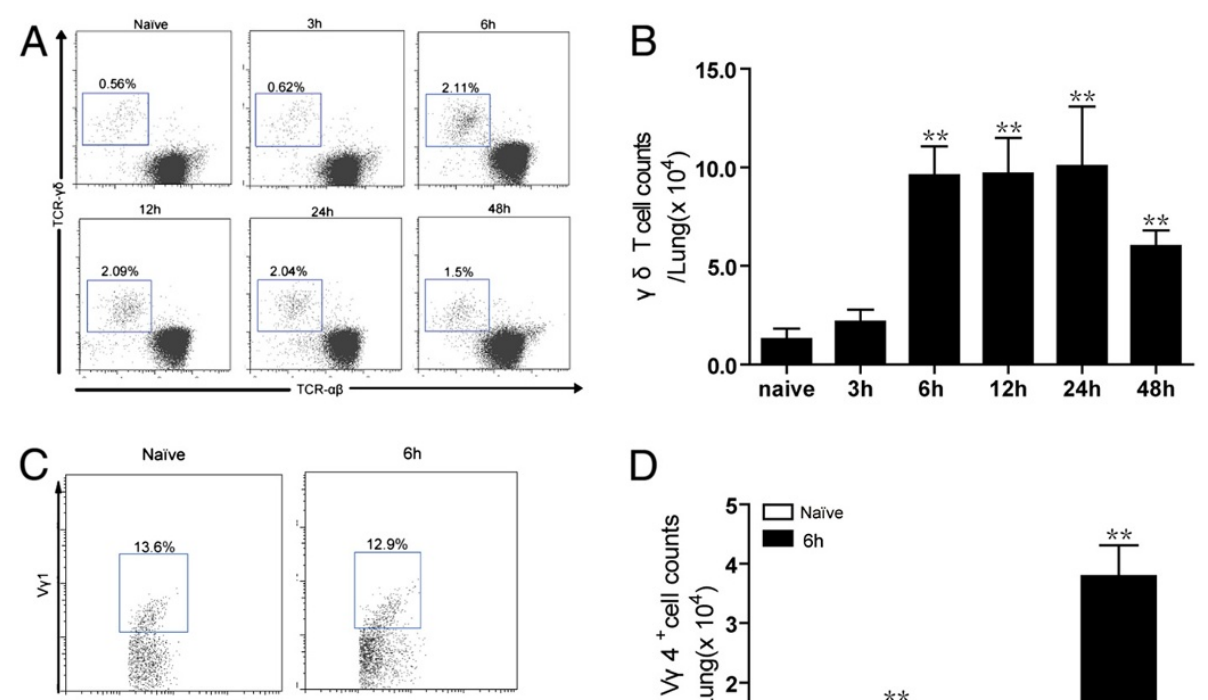

\section{D}
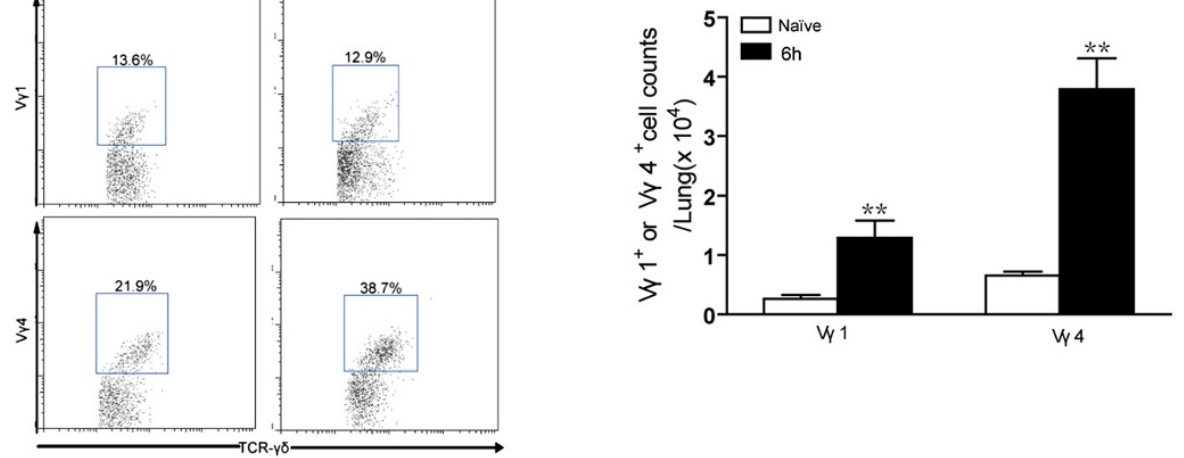

Figure 1 Pulmonary $\gamma \delta$ T cells are increased following S. aureus infection. C57BL/6 mice were intranasally infected with SA 75 strain or PBS as mocked infection and sacrificed at different time post infection. (A) Pulmonary leukocytes were isolated and TCRa $\beta^{-} \mathrm{TCR} \delta^{+}$cells were detected by flow cytometry. Cells were gated on $\mathrm{CD}^{+}$cells. Representative flow cytometry dot-plots were shown. Numbers represent the percentage of $\gamma \delta$ T cells. (B) Absolute numbers of $\gamma \delta$ T cells in the total lung at various time points following S.aureus challenge were shown. (C) The expression of $V_{\gamma} 1$ and $V_{\gamma} 4$ chains on gated $C D 3^{+} T C R a \beta^{-} T C R \gamma \delta^{+}$lung cells were analyzed by flow cytometry. Numbers indicate percentages of $V_{\gamma} 1^{+}$or $V_{Y} 4^{+} \gamma \delta T$ cells. Representative flow cytometry dot-plots were shown. (D) Absolute numbers of $\mathrm{V}_{\gamma} 1^{+}$or $\mathrm{V}_{\mathrm{Y}} 4^{+} \gamma \delta \mathrm{T}$ cells in naive and $6 \mathrm{~h}$ post-infection lungs. Bars represent mean \pm SEM of 3-6 mice/time from three independent experiments. ${ }^{* *} p<0.01$ versus naive control.

\section{IL-17-producing $\gamma \delta T$ cells provided early responses to $S$. aureus challenge}

IL-17 is an important proinflammatory cytokine involved in the migration and activation of neutrophils, and it is mainly produced by $\gamma \delta \mathrm{T}$ cell in several infectious diseases $[14,15]$. To examine whether $\gamma \delta$ T cells produce IL-17 after S.aureus infection, production of IFN- $\gamma$ and IL-17 by $\gamma \delta$ T cells was analyzed by intracellular staining. We observed that the percentage of IFN $-\gamma^{+}$cells among $\gamma \delta \mathrm{T}$ cells was slightly decreased in lungs at $6 \mathrm{~h}$ postinfection. Whereas, accounting for the increased total $\gamma \delta$ $\mathrm{T}$ cells number, there was a significant increase of absolute number of IFN- $\gamma^{+} \gamma \delta$ T cells following S.aureus challenge (Figure 5B). Meanwhile, a significant increased proportion of IL- $17^{+} \gamma \delta$ T cells were also observed. Consistently, their absolute number almost increased up to 30 -fold at $6 \mathrm{~h}$ post infection (Figure 5A\&5B). Further analysis by flow cytometry showed that $\gamma \delta \mathrm{T}$ cells are the primary source for IL-17 in the lungs of S.aureus infected mice. About $60 \%$ of lung IL-17-producing T cells was TCR- $\delta^{+}$, and few $\mathrm{CD} 4^{+}$T cells expressed IL-17 (Figure $5 \mathrm{C}$ ). We next analyzed if $\gamma \delta$ T cell-deficient mice show any changes on the level of IL-17 after S.aureus infection. WT mice displayed an early burst of IL-17 expression, which increased at 6 hours and was sustained at a high level at all subsequent time points evaluated. In contrast, a significantly lower expression of IL-17 mRNA in the lungs of $\gamma \delta \mathrm{T}$ cell-deficient infected mice was observed from 6 hours post-infection onwards. In addition, $\gamma \delta \mathrm{T}$ cell-deficient mice exhibited delayed onset of IL-17 induction in infected lungs (Figure 5C). Together, these data implied that lung IL-17-producing $\gamma \delta \mathrm{T}$ cell provide early responses to bacteria challenge and deficiency of $\gamma \delta$ T cells results in a defect of IL-17 induction after S.aureus infection.

\section{Decreased expression of neutrophil-inducing} cytokine/chemokine in infected lungs of TCR- $\delta^{-/-}$mice

IL-17 regulates the production of CXC chemokines such as murine MIP-2/CXCL2, KC/CXCL1, which are necessary for neutrophil recruitment. Also, IL-17 participates in the induction of cytokines required for neutrophil differentiation and activation, including GM-CSF, IL-6 and TNF- $\alpha$ [16]. To further elucidate the relationship 

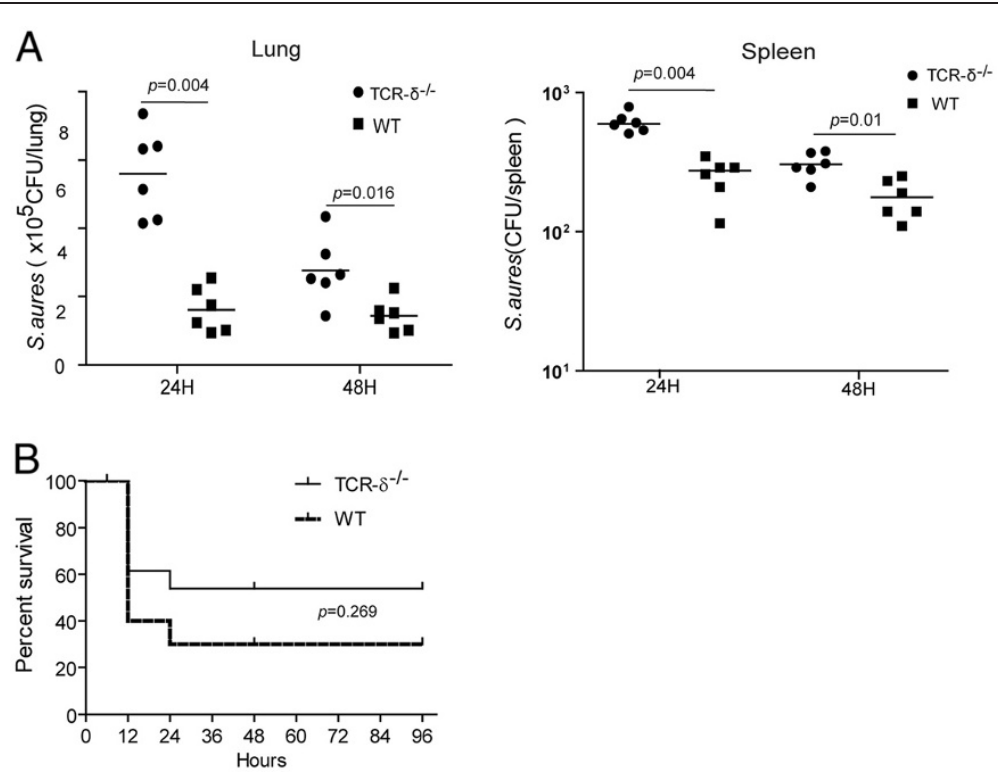

Figure 2 Deficiency of $\gamma \delta$ T cells leads to increased bacteria burden. (A) Mice were inoculated with sub-lethal SA 75 strain $\left(5 \times 10^{8} \mathrm{CFU}\right.$, $n=6 /$ group/time point). Bacterial burden in lung and spleen were determined at 24 and 48 hours following pulmonary infection with S.aureus. Each dot represents the bacterial count of the respective organ of a single animal. The line represents the median of each group. (B) Survival curve of WT $(n=8)$ and TCR- $\delta^{-/-}(n=9)$ mice after intranasally inoculated with $5 \times 10^{9}$ CFU bacteria. $p=0.269$ for infected TCR- $\delta^{-/-}$versus WT mice.

between the impaired neutrophil recruitment and decreased IL-17 production in TCR- $\delta^{-1-}$ mice, we compared the expression of these neutrophil-inducing cytokines/chemokines in the lungs of S.aureus infected TCR- $\delta^{-/-}$and WT mice at $6 \mathrm{~h}$ post-infection. As shown in Figure 6, KC and MIP-2 production were severely impaired in the lungs of infected TCR- $\delta^{-/-}$mice in comparison to WT mice. Similarly, the expression of GM-CSF, IL-6, and TNF- $\alpha$ were also significantly reduced in the TCR- $\delta^{-1-}$ mice, compared with those in the wild-type mice. These results implied that the impaired neutrophil recruitment in the absence of $\gamma \delta \mathrm{T}$ cell was caused, to some extent, by the decreased expression of neutrophil-inducing cytokine/chemokine at the early stage of infection.

\section{Discussion}

In this study, we investigated the role of $\gamma \delta \mathrm{T}$ cells in the early host response to $S$. aureus infection. A significant accumulation of $\gamma \delta \mathrm{T}$ cells at lungs was observed as early as $6 \mathrm{~h}$ post-infection. The local expansion of resident $\gamma \delta \mathrm{T}$ cells and/or recruitment from the peripheral circulation may be responsible for the accumulation of $\gamma \delta \mathrm{T}$ cells in the lungs following S.aureus infection. In murine lungs, ${\mathrm{V} \gamma 1^{+}}^{+}$and ${\mathrm{V} \gamma 4^{+}}^{+} \delta \mathrm{T}$ cells subsets are two major subsets $[10,13]$. Generally, ${\mathrm{V} \gamma 4^{+}}^{+} \delta \mathrm{T}$ cells are the dominant population of lung $\gamma \delta \mathrm{T}$ cells and $\mathrm{V} \gamma 1^{+} \mathrm{T}$ cells are mainly located in lymphoid organs. Both subsets are involved in the immune response to various pulmonary infectious pathogens. For example, in the lungs of BCG-infected mice, substantial $\mathrm{V} \gamma 1^{+}$and ${\mathrm{V} \gamma 4^{+}}^{+} \delta$ T cells accumulate at the site of infection. Concomitant with the expansion of pulmonary $\mathrm{V}_{\gamma} 4^{+} \gamma \delta \mathrm{T}$ cells, large amounts of $\mathrm{V} \gamma 1^{+} \gamma \delta \mathrm{T}$ cells were recruited to the lungs of infected mice [17]. Also, both subsets of $\gamma \delta$ T cells were significantly increased following $S$. pneumoniae challenge [13]. But conversely, $\mathrm{V}_{\gamma} 4^{+} \gamma \delta \mathrm{T}$ cells contributed to the increased size of $\gamma \delta \mathrm{T}$ cells and ablation of ${\mathrm{V} \gamma 4^{+}}^{+} \delta \mathrm{T}$ cells attenuated neutrophil recruitment and impaired host protection [8]. Similarly, in our present study, both $\mathrm{V} \gamma_{\gamma} 1^{+}$and $\mathrm{V} \gamma^{+} 4^{+} \gamma \delta \mathrm{T}$ cells were increased in the lungs. Whereas ${\mathrm{V} \gamma 4^{+}}^{+} \delta \mathrm{T}$ cells were the dominant population of the increased pulmonary $\gamma \delta$ T cells at the early phase of S.aureus infection. Compared with lung, no significant increase of $\gamma \delta$ T cells in the spleen and draining lymph node of infected mice was observed (data not shown). We presume that accumulated $\gamma \delta \mathrm{T}$ cells were restricted to the lung at the early phase of S.aureus infection. Of note, approximately $47 \%$ of $\gamma \delta \mathrm{T}$ cells expressing other $\mathrm{V}_{\gamma}$ genes were unidentified due to limited availability of antibodies in our lab. These cells may include remaining pulmonary $\mathrm{V}_{\gamma} 2^{+} \gamma \delta \mathrm{T}$ cells and/or $\mathrm{V}_{\gamma} 6^{+} \gamma \delta \mathrm{T}$ cells, as suggested by previous studies [17]. These subsets have not been well defined in the course of pulmonary infection. To determine whether $\gamma \delta$ T-cell subsets have a differential role in the immune response to S.aureus-induced pneumonia will require further studies.

$\gamma \delta \mathrm{T}$ cells exert different influences on the host response in a variety of infection models. In some 
Figure 3 The absence of $\gamma \delta$ T cells leads to less severe lung lesions after $\mathbf{S}$. aureus-induced pneumonia infection. Lungs were harvested 12 hours following S. aureus challenge. (A) Representative $\mathrm{H}$-E staining of lung sections from mock-infected control mice, TCR$\delta^{-1-}$ mice and WT mice and (B) Histological scores of pneumonia in WT and TCR- $\delta^{-/-}$mice ( $n=8 /$ group) were shown.

experimental animal models of pulmonary infections, $\gamma \delta$ $\mathrm{T}$ cells were demonstrated to be protective against pathogens infection, such as M. tuberculosis [12], Klebsiella pneumoniae [8,11], Nocardia asteroides [18] and Cryptococcus neoformans [19]. In these infections the absence of $\gamma \delta$ T cells rendered mice more susceptible to pathogen infection. In contrast, other studies reported that $\gamma \delta \mathrm{T}$ cells were not necessary to the host defense against microbial infection and even harmful to the host. Following challenge with Candida albicans [20] or Salmonella choleraesuis [21], $\gamma \delta \mathrm{T}$ cell knock out mice were resistant to infection and showed an accelerated pathogens clearance. So these data indicate that $\gamma \delta$ T cell might exert diverse function in different infection disease depending on the unique of bacterial infection. In our current study, compared with wild-type mice, $\gamma \delta \mathrm{T}$ cell deficient mice show no significant change in survival. But the absence of these cells led to attenuated bacterial clearance. It appears that accumulated $\gamma \delta \mathrm{T}$ cells in the lungs might contribute to the host protective responses against S.aureus infection.

Contrastingly, regardless of bacteria load, we found that mutant mice exhibited less severe pulmonary lesions at the early stage of infection. Since neutrophils are responsible for the acute inflammation response to most infection in the lung, we next detected the neutrophils infiltration. The results showed that the infiltration of neutrophils in the lungs was less in TCR $\delta^{-/-}$mice. It is well known that recruited neutrophils provide the first line of defense against many infections by ingesting the foreign invaders and limiting the pathogen survival and dissemination [4]. It is reported that depletion of neutrophils led to a fatal defect in murine pulmonary S.aureus clearance [5]. On the contrary, neutrophils have also been implicated in the pathology of many inflammatory conditions by releasing oxidants and hydrolytic enzymes [22]. Earlier studies reported that respiratory syncytial virus (RSV) could enhance neutrophils infiltration to the lung and the activated neutrophils significantly augmented RSV induced damage [23]. And pulmonary neutrophil infiltration is an insidious killer in polymicrobial sepsis, in patients with an acute bacterial infection, primed neutrophils with increased oxidative product formation contributed to the damage of pulmonary vascular endothelium during bacteremia [24]. Previous studies also reported that neutrophil recruitment during S. aureus USA300-induced acute pneumonia led to 

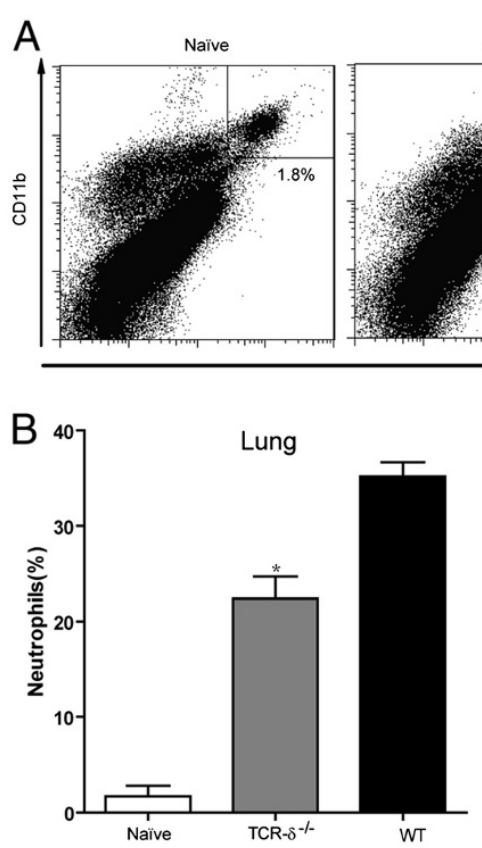

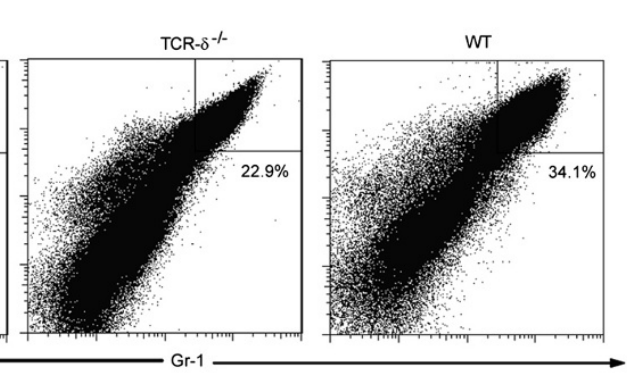

C

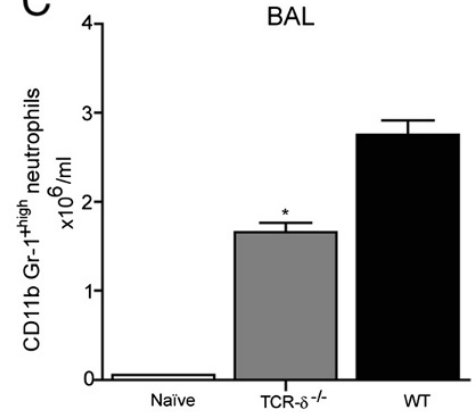

Figure $4 \gamma \delta$ T cell-deficient mice have markedly impaired neutrophil recruitment during S. aureus-induced pneumonia. WT and TCR- $\delta^{-/-}$ mice (3-6 mice/group) were intranasally inoculated with S.aureus. Cells were prepared from the lung at $6 \mathrm{~h}$ post infection and neutrophils were detected by flow cytometry. The representative dot-plots $(\mathbf{A})$ and statistical results $(\mathbf{B})$ were shown $(n=6)$. (C) Lungs of TCR- $\delta^{-1-}$ mice and WT were lavaged and the number of neutrophils in BAL fluid was determined by flow cytometry. ${ }^{*} p<0.05$ versus WT.

serious lung inflammation and injury, which was associated with the overwhelming release of cytotoxic granule contents [25]. Similarly, other reports demonstrated that the accumulated neutrophils at the site of infection may exacerbate the severity of $S$. aureus wound infection [26]. Our present study also suggested that the reduced infiltration of neutrophils might be responsible for both the increased bacterial dissemination and the less tissue damage in TCR $\delta^{-/-}$mice.

Consistent with the decreased recruitment of neutrophils to S.aureus infection in the TCR $\delta^{-1-}$ mice, we also found decreased KC, MIP-2, GM-CSF, TNF-a, IL-6 and IL-17 in lung. These factors are known to regulate the neutrophil infiltration and their reduction might contribute to the less neutrophil infiltration. IL-17 is worthy of note among these factors, since it has been shown to be a crucial regulator of the migration and activation of neutrophils and also secreted by the $\gamma \delta$ T cells. $\gamma \delta$ T cells are responsible for much of this rapid cytokine production in the earliest stages of an inflammatory response [27]. Some groups reported that IL-17A-producing cells were essential for vaccine efficacy against systemic S. aureus infection [28,29]. Previous study reported that IL-17 deficient mice displayed impaired host defense against mucoepithelial infection by $S$. aureus [30]. Similarly, Kudva et al. described the impact of IL-17-mediated immune response against $S$. aureus pneumonia. Mice deficient in IL-17R showed impaired bacteria clearance from the lung [31]. In this study, we found that the main source of IL-17 in S.aureus-infected lung was $\gamma \delta$ T cells and depletion of $\gamma \delta$ T cells results in the reduced IL-17 expression in the early phase of infection. These results implied that the impaired neutrophil accumulation in $\gamma \delta \mathrm{T}$ cell-deficient mice was likely caused, at least in part, by the decreased production of IL-17 at the site of infection. Nevertheless, no direct evidence of the involvement of IL-17-producing $\gamma \delta$ T cells was presented. We cannot exclude the possibility that other products of $\gamma \delta \mathrm{T}$ cells may have effects on the early neutrophil recruitment. The role for IL-17producing $\gamma \delta$ T cells in the S.aureus-induced pneumonia awaits further study.

\section{Conclusions}

In conclusion, $\gamma \delta \mathrm{T}$ cells accumulated in the lungs after S.aureus infection, which was the primary source of IL17 at the early phase of infection. Its presence is beneficial for bacteria clearance but also contributes the early acute inflammation and tissue injury in lung. The function of $\gamma \delta$ T cells in S. aureus-induced pneumonia might be mediated by neutrophil infiltration. Overall, our findings provide insight into the complex innate immune system in early pulmonary S.aureus infection. Further study is required to elucidate the detailed mechanisms 

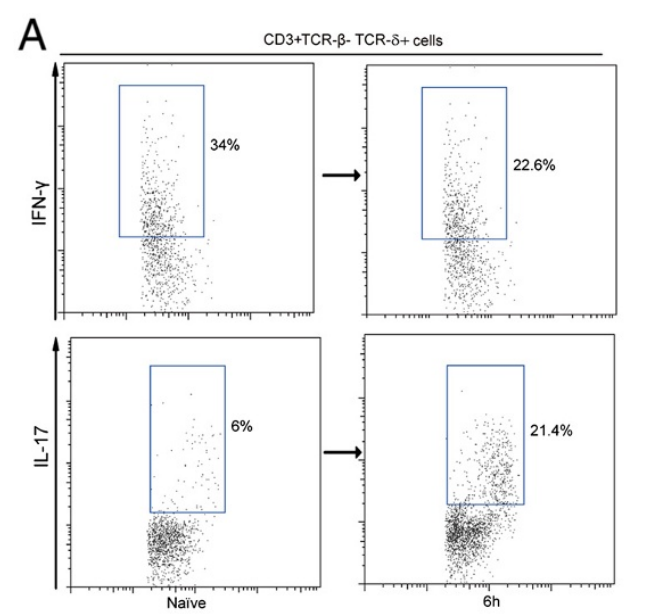

\section{B}
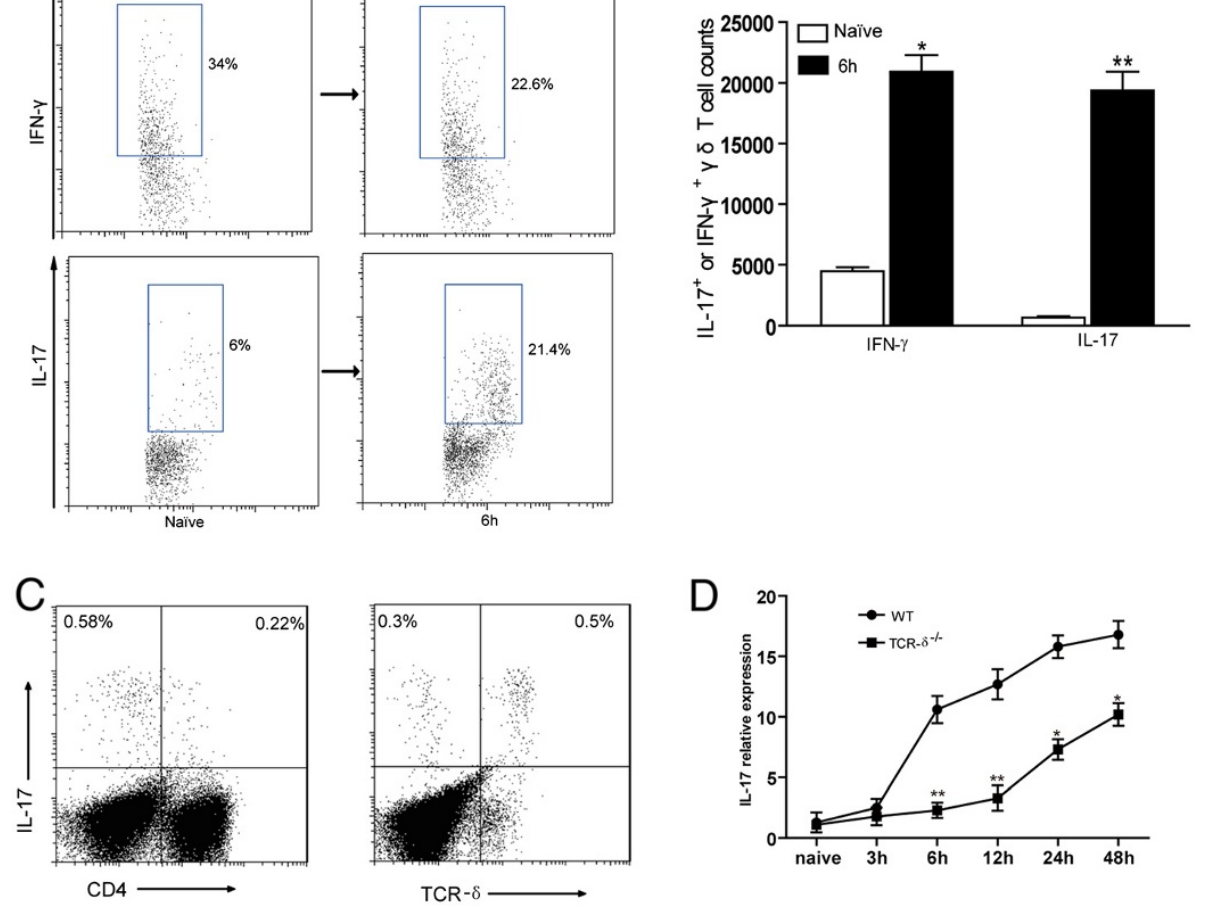

Figure 5 Increase of IL-17-producing $\gamma \delta$ cells following S. aureus challenge and reduced IL-17 induction in $\gamma \delta \mathrm{T}$ cells deficient mice. Total lung cells were isolated from naive or S.aureus-challenged mice at $6 \mathrm{~h}$ post-infection and IL-17 and IFN- $\gamma$ producing $\gamma \delta^{+} T$ cell were detected by intracellular staining. Cells were gated on $C D 3^{+} \mathrm{TCRa}^{-} \mathrm{TCR} \gamma \delta^{+}$cells. (A) The representative dot-plots were shown. Numbers indicate

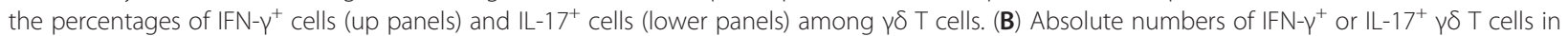
naive and $6 \mathrm{~h}$ post-infection lungs. (C) The representative dot plots showed the percentages of IL-17 producing cells in lung $\mathrm{CD}^{+} \mathrm{T}$ lymphocytes. Numbers in the quadrant indicate the percentage of cells. (D) IL-17A mRNA expression in the lungs following S. aureus challenge was determined by quantitative real-time PCR. Data are expressed as the mean \pm SEM of 3-6 mice/time from three independent experiments. ${ }^{*} p<0.05$ or ${ }^{* *} p<0.01$ versus WT mice.

by which $\gamma \delta \mathrm{T}$ cells control the bacteria clearance and facilitate the tissue damage, which might be helpful to find a way to regulate the $\gamma \delta \mathrm{T}$ cell to benefit bacteria clearance and avoid the tissue damage.

\section{Methods}

\section{Mice}

Six- to 8-wk-old female C57BL/6 mice were purchased from the Experimental Animal Center of the Third Military Medicine University. B6.129P2-Tcrd ${ }^{\text {tm1Mom}} / \mathrm{J}$ (B6 TCR $-\delta^{-/}$) mice were kindly provided by Dr. Zhinan Yin (College of Life Sciences, Nankai University, Tianjin, China). All mice were housed in specific pathogen-free facilities. All experiments were approved by the Animal Ethical and Experimental Committee of Third Military Medical University.

\section{Bacteria strains and mouse pneumonia model}

The mouse model of $S$. aureus pneumonia was established as previously described [7]. Briefly, a clinical isolate of the $S$. aureus SA75 strain was cultured in
Mueller Hinton Agar (MHA) for 24 hours prior to inoculation into Mueller Hinton broth which was then incubated overnight. Then the overnight culture was diluted 1:100 into fresh Mueller Hinton broth (MHB). Bacteria were grown at $200 \mathrm{rpm}$ at $37^{\circ} \mathrm{C}$ to an optical density at $660 \mathrm{~nm}$ of 0.5 . Cells were collected and washed twice with PBS. All mice were anesthetized with isoflurane and inoculated intranasally with $5 \times 10^{8}$ or $5 \times 10^{9} \mathrm{CFU}$ of $S$. aureus SA75. Animals were held upright for $1 \mathrm{~min}$ post-inoculation. Mock-infected control mice were inoculated intranasally with sterile PBS. Mice were either sacrificed at pre-determined time points or assessed for survival for $48 \mathrm{~h}$.

\section{Isolation of pulmonary leukocytes}

Pulmonary leukocytes were isolated as previously described [19]. Briefly, the whole lungs were removed aseptically and cut into small pieces and incubated in RPMI 1640 (Hyclone) containing 20U/ml collagenase type I and $1 \mu \mathrm{g} / \mathrm{ml}$ DNase I (Sigma-Aldrich)for $1 \mathrm{~h}$ at $37^{\circ} \mathrm{C}$ under continuous rotation. And the lung portions 


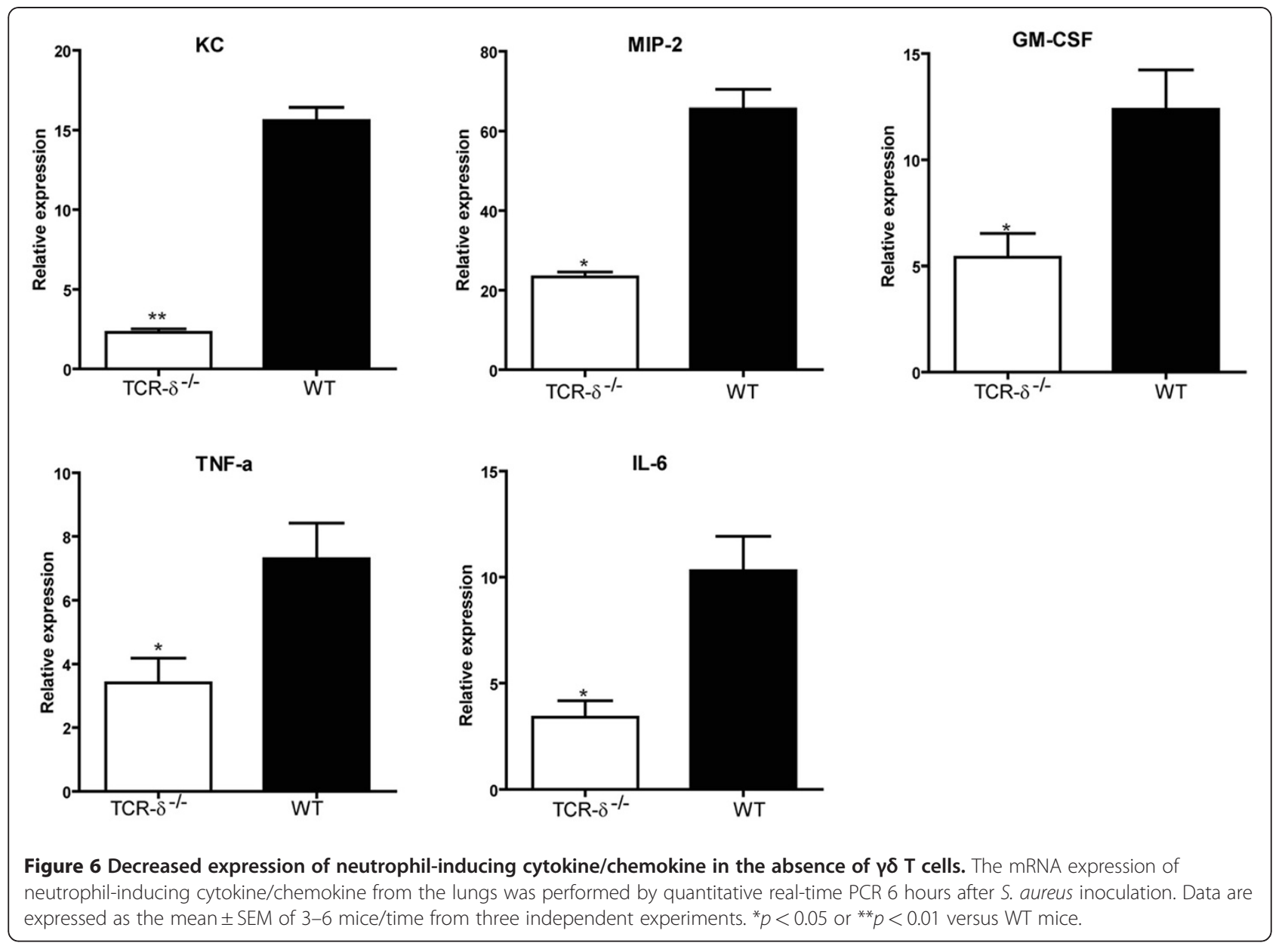

were crushed through $40 \mu \mathrm{m}$ meshed steel sieves. The total cell pellet was resuspended in $3 \mathrm{ml}$ of $40 \%$ (v/v) Percoll (Pharmacia, Uppsala, Sweden) and layered onto $3 \mathrm{ml}$ of $80 \%(\mathrm{v} / \mathrm{v})$ Percoll. After centrifugation at $600 \mathrm{x} \mathrm{g}$ for $20 \mathrm{~min}$ at room temperature, the cells at the interface were collected and washed in PBS. All isolated cells were enumerated and resuspended in RPMI 1640. Total viable cell counts were determined by trypan blue exclusion.

\section{Bronchoalveolar lavage (BAL)}

Bronchoalveolar lavage (BAL) was performed as previously described [8]. Briefly, the chest was opened and the trachea was exposed through a midline incision and cannulated with a sterile 22-gauge Abbocath-T catheter and BAL was performed by instillation of two $0.5-\mathrm{ml}$ aliquots of sterile saline. Approximately $0.8 \mathrm{ml}$ of BAL fluid was retrieved per mouse.

\section{Bacterial counts}

Mice were sacrificed at $24 \mathrm{~h}$ and $48 \mathrm{~h}$ postinfection and the levels of bacterial load were determined by preparing lung and spleen homogenates in PBS and plating serially dilutions on MHA. After $18 \mathrm{~h}$ of incubation at $37^{\circ} \mathrm{C}$, colony forming units were calculated by standard plate counting and presented as CFU/organ. BAL was collected and plated 10-fold serial dilutions on MHA. Colonies were determined as $\mathrm{CFU} / \mathrm{ml}$.

\section{Histopathology}

Lung tissue was fixed with paraformaldehyde and embedded in paraffin, sectioned, and stained with hematoxylin and eosin (H\&E). A double-blind histological analysis was performed to examine the sections from each lung of mock-infected and infected mice, and each section was given a score of $0-4$ (no abnormality to most severe pneumonia) according to established criteria [5].

\section{Flow cytometry}

FACS was performed to analyze cell surface marker and intracellular cytokine expression. Briefly, Lung cells were isolated as mentioned above and stimulated for $6 \mathrm{~h}$ with PMA (50 ng/ml; Sigma-Aldrich, St. Louis, MO), ionomycin $(1 \mu \mathrm{g} / \mathrm{ml}$; Sigma-Aldrich) and Golgistop (BD Pharmingen, San Diego, CA). The cells were blocked with anti-CD16/32 
antibody (clone 2.4 G2; BD Biosciences) on ice for $15 \mathrm{~min}$, stained for $30 \mathrm{~min}$ with the following specific Abs: FITC-anti- $\gamma \delta$ TCR(clone GL3), PECy5.5-anti-TCR $\beta$ (clone H57-597) and PerCP-Cy5.5-anti-CD8 (clone 53-6.7) were purchased from eBioscience(San Diego, CA), FITCanti-GR-1(clone RB6-8 C5), APC-Cy7-anti-CD3 (clone 17A2), PerCP-Cy5.5-anti-CD4 (clone RM4-5) and PerCPCy5.5-anti-CD11b (clone M1/70) were from Biolegend, PE-anti-TCR V V1 (clone 2.11) and APC anti- TCR V Y4 (clone UC3) were from Tianjin Sungene (Tianjin, China). Intracellular cytokine staining was performed after fixation and permeabilization, using Perm/Wash solution (BD Biosciences). The cells were then separately stained intracellularly with PE-Cy7-anti-IFN- $\gamma$ (XMG1.2) and PE-anti-IL -17A (TC11-18 H10) (BD Biosciences). Samples were acquired on FACSCanto II (BD Biosciences). Data were analyzed with Flowjo software or FACSDiva software (BD Biosciences).

\section{Lung RNA isolation and Real-time PCR}

Total lung RNA was isolated using Trizol (Gibco), according to the manufacturer's instructions. DNA was removed from RNA preparations with DNase I digestion (Invitrogen) for $30 \mathrm{~min}$ at $37^{\circ} \mathrm{C}$. And samples were reverse-transcribed to cDNA using the PrimeScript ${ }^{\circledR}$ RT Master Mix (Takara) according to the manufacturers' instructions. Real-time PCR was performed on the iQ5 apparatus (BioRad, Hercules, USA). The cycle number at which the various transcripts were detectable, referred to as the threshold cycle (Ct), was compared with that of $\beta 2-\mathrm{M}$ and referred to as $\Delta \mathrm{Ct}$. The relative gene expression was expressed as fold change calculated by the ${ }^{\Delta \Delta} \mathrm{Ct}$ method. The expression of IL-17A was determined by the TaqMan method using primers and probes previously reported [32]. The mRNA of keratinocyte-derived chemokine (KC), macrophage inflammatory protein 2 (MIP-2), GM-CSF, tumor necrosis factor alpha (TNF- $\alpha$ ) and IL- 6 were measured by SYBR Green Realtime PCR Master Mix (Toyobo) using the previously described primers [15]. Mouse $\beta 2-\mathrm{M}$ served as the normalizer.

\section{Statistical analysis}

All data are expressed as means \pm standard errors of the means (SEM). The statistical analysis software used was SPSS Version13.0. Normally distributed data were analyzed using Student's $t$-test. Otherwise, the non-parametric Mann-Whitney test was used. Survival studies were analyzed using Kaplan-Meier testing. A $P$ value of $<0.05$ was considered to represent a statistically significant difference. The data were from at least three independent experiments.

\section{Competing interests}

The authors declare that they have no competing interests.

\section{Authors' contributions}

Conceived and designed the experiments: QZ, YS and TL. Performed the experiments: PC, WZ, YZ, LP, and JZ. Analyzed the data: PC, TL, XM and YS. Contributed reagents/materials/analysis tools: PC, TL, WZ, ZY, XM and GG. Wrote the paper: PC, TL, YS, QZ. All authors read and approved the final manuscript.

\section{Acknowledgements}

This work was supported by National Natural Science Foundation of China (81000715) and Chongqing Science and Technology Commission (2008BB5114).

\section{Author details}

'Department of Clinical Microbiology and Immunology, Faculty of Medical Laboratory Science, Third Military Medical University and National Engineering Technological Research Center of Immunological Biologicals, Chongqing 400038, China. ${ }^{2}$ Department of Pharmacology, College of Pharmacy, The Third Military Medical University, Chongqing, China. ${ }^{3}$ College of Life Sciences, Nankai University, Tianjin, China.

Received: 19 March 2012 Accepted: 20 June 2012

Published: 9 July 2012

\section{References}

1. Moran GJ, Krishnadasan A, Gorwitz RJ, Fosheim GE, McDougal LK, Carey RB, Talan DA: Methicillin-resistant S. aureus infections among patients in the emergency department. N Engl J Med 2006, 355:666-674.

2. Klevens RM, Morrison MA, Nadle J, Petit S, Gershman K, Ray S, Harrison LH, Lynfield R, Dumyati G, Townes JM, et al: Invasive methicillin-resistant Staphylococcus aureus infections in the United States. JAMA 2007, 298:1763-1771.

3. Howard LS, Sillis M, Pasteur MC, Kamath AV, Harrison BD: Microbiological profile of community-acquired pneumonia in adults over the last 20 years. J Infect 2005, 50:107-113.

4. Rigby KM, Deleo FR: Neutrophils in innate host defense against Staphylococcus aureus infections. Semin Immunopathol 2012, 34:237-259.

5. Robertson CM, Perrone EE, McConnell KW, Dunne WM, Boody B, Brahmbhatt T, Diacovo MJ, Van Rooijen N, Hogue LA, Cannon CL, et al: Neutrophil depletion causes a fatal defect in murine pulmonary Staphylococcus aureus clearance. J Surg Res 2008, 150:278-285.

6. Michailova L, Stoitsova S, Markova N, Kussovski V, Jordanova M, Dimova I: Interaction of alveolar macrophages with Staphylococcus aureus and induction of microbial L-forms during infection in rats. Int I Med Microbiol 2000, 290:259-267.

7. Martin FJ, Parker D, Harfenist BS, Soong G, Prince A: Participation of $\mathrm{CD} 11 \mathrm{c}+$ leukocytes in the host response to MRSA infection of the lung. Infect Immun 2011, 79:1898-1904.

8. Nakasone C, Yamamoto N, Nakamatsu M, Kinjo T, Miyagi K, Uezu K, Nakamura K, Higa F, Ishikawa H: O'Brien R L, et al: Accumulation of gamma/delta T cells in the lungs and their roles in neutrophil-mediated host defense against pneumococcal infection. Microbes Infect 2007, 9:251-258.

9. Kirby AC, Newton DJ, Carding SR, Kaye PM: Pulmonary dendritic cells and alveolar macrophages are regulated by gammadelta $T$ cells during the resolution of S. pneumoniae-induced inflammation. J Pathol 2007, 212:29-37.

10. Wands JM, Roark CL, Aydintug MK, Jin N, Hahn YS, Cook L, Yin X, Dal Porto J, Lahn M, Hyde DM, et al: Distribution and leukocyte contacts of gammadelta T cells in the lung. J Leukoc Biol 2005, 78:1086-1096.

11. Moore TA, Moore BB, Newstead MW, Standiford TJ: Gamma delta-T cells are critical for survival and early proinflammatory cytokine gene expression during murine Klebsiella pneumonia. J Immunol 2000, 165:2643-2650.

12. Lockhart $\mathrm{E}$, Green AM, Flynn JL: IL-17 production is dominated by gammadelta T cells rather than CD4 T cells during Mycobacterium tuberculosis infection. J Immunol 2006, 177:4662-4669. 
13. Kirby AC, Newton DJ, Carding SR, Kaye PM: Evidence for the involvement of lung-specific gammadelta $T$ cell subsets in local responses to Streptococcus pneumoniae infection. Eur J Immunol 2007, 37:3404-3413.

14. Cho JS, Pietras EM, Garcia NC, Ramos Rl, Farzam DM, Monroe HR, Magorien JE, Blauvelt A, Kolls JK, Cheung AL, et al: IL-17 is essential for host defense against cutaneous Staphylococcus aureus infection in mice. J Clin Invest 2010, 120:1762-1773.

15. Umemura M, Yahagi A, Hamada S, Begum MD, Watanabe H, Kawakami K, Suda T, Sudo K, Nakae S, Iwakura Y, Matsuzaki G: IL-17-mediated regulation of innate and acquired immune response against pulmonary Mycobacterium bovis bacille Calmette-Guerin infection. J Immunol 2007, 178:3786-3796.

16. Korn T, Bettelli E, Oukka M, Kuchroo VK: IL-17 and Th17 Cells. Annu Rev Immunol 2009, 27:485-517.

17. Dieli F, Ivanyi J, Marsh P, Williams A, Naylor I, Sireci G, Caccamo N, Di Sano C, Salerno A: Characterization of lung gamma delta T cells following intranasal infection with Mycobacterium bovis bacillus Calmette-Guerin. J Immunol 2003, 170:463-469

18. Tam S, King DP, Beaman BL: Increase of gammadelta T lymphocytes in murine lungs occurs during recovery from pulmonary infection by Nocardia asteroides. Infect Immun 2001, 69:6165-6171.

19. Uezu K, Kawakami K, Miyagi K, Kinjo Y, Kinjo T, Ishikawa H, Saito A: Accumulation of gammadelta $T$ cells in the lungs and their regulatory roles in Th1 response and host defense against pulmonary infection with Cryptococcus neoformans. J Immunol 2004, 172:7629-7634.

20. Wormley FL Jr, Steele C, Wozniak K, Fujihashi K, McGhee JR, Fidel PL Jr: Resistance of T-cell receptor delta-chain-deficient mice to experimental Candida albicans vaginitis. Infect Immun 2001, 69:7162-7164.

21. Emoto M, Nishimura H, Sakai T, Hiromatsu K, Gomi H, Itohara S, Yoshikai Y: Mice deficient in gamma delta T cells are resistant to lethal infection with Salmonella choleraesuis. Infect Immun 1995, 63:3736-3738.

22. Butterfield TA, Best TM, Merrick MA: The dual roles of neutrophils and macrophages in inflammation: a critical balance between tissue damage and repair. J Athl Train 2006, 41:457-465.

23. Wang SZ, Xu H, Wraith A, Bowden JJ, Alpers JH, Forsyth KD: Neutrophils induce damage to respiratory epithelial cells infected with respiratory syncytial virus. Eur Respir J 1998, 12:612-618.

24. Bass DA, Olbrantz P, Szejda P, Seeds MC, McCall CE: Subpopulations of neutrophils with increased oxidative product formation in blood of patients with infection. J Immunol 1986, 136:860-866.

25. Diep BA, Chan L, Tattevin P, Kajikawa O, Martin TR, Basuino L, Mai TT, Marbach H, Braughton KR, Whitney AR, et al: Polymorphonuclear leukocytes mediate Staphylococcus aureus Panton-Valentine leukocidin-induced lung inflammation and injury. Proc Natl Acad Sci U S A 2010, 107:5587-5592.

26. McLoughlin RM, Solinga RM, Rich J, Zaleski KJ, Cocchiaro JL, Risley A Tzianabos AO, Lee JC: CD4+ T cells and CXC chemokines modulate the pathogenesis of Staphylococcus aureus wound infections. Proc Natl Acad Sci U S A 2006, 103:10408-10413.

27. Duan J, Chung H, Troy E, Kasper DL: Microbial colonization drives expansion of IL-1 receptor 1-expressing and IL-17-producing gamma/ delta T cells. Cell Host Microbe 2010, 7:140-150.

28. Narita K, Hu DL, Mori F, Wakabayashi K, Iwakura Y, Nakane A: Role of interleukin-17A in cell-mediated protection against Staphylococcus aureus infection in mice immunized with the fibrinogen-binding domain of clumping factor A. Infect Immun 2010, 78:4234-4242.

29. Lin L, Ibrahim AS, Xu X, Farber JM, Avanesian V, Baquir B, Fu Y, French SW, Edwards JE Jr, Spellberg B: Th1-Th17 cells mediate protective adaptive immunity against Staphylococcus aureus and Candida albicans infection in mice. PLoS Pathog 2009, 5:e1000703.

30. Ishigame H, Kakuta S, Nagai T, Kadoki M, Nambu A, Komiyama Y, Fujikado N, Tanahashi Y, Akitsu A, Kotaki H, et al: Differential roles of interleukin-17A and $-17 \mathrm{~F}$ in host defense against mucoepithelial bacterial infection and allergic responses. Immunity 2009, 30:108-119.

31. Kudva A, Scheller EV, Robinson KM, Crowe CR, Choi SM, Slight SR, Khader SA Dubin PJ, Enelow RI, Kolls JK, Alcorn JF: Influenza A inhibits Th17-mediated host defense against bacterial pneumonia in mice. J Immunol 2011, 186:1666-1674.

32. Shi Y, Liu XF, Zhuang Y, Zhang JY, Liu T, Yin Z, Wu C, Mao XH, Jia KR, Wang FJ, et al: Helicobacter pylori-induced Th17 responses modulate Th1 cell responses, benefit bacterial growth, and contribute to pathology in mice. $\mathrm{J}$ Immunol 2010, 184:5121-5129. doi:10.1186/1471-2172-13-38

Cite this article as: Cheng et al:: Role of gamma-delta T cells in host response against Staphylococcus aureus-induced pneumonia. BMC Immunology 2012 13:38.

\section{Submit your next manuscript to BioMed Central and take full advantage of:}

- Convenient online submission

- Thorough peer review

- No space constraints or color figure charges

- Immediate publication on acceptance

- Inclusion in PubMed, CAS, Scopus and Google Scholar

- Research which is freely available for redistribution 